\title{
Real-time precision opto-control of chemical processes in live cells
}

\section{Authors:}

Matthew G. Clarkㄹ, Gil Gonzalez¹, Jesus Aldana-Mendoza ${ }^{1}$, Mark S. Carlsen ${ }^{1}$, Gregory Eakins $^{1}$, and Chi Zhang ${ }^{1,2,3 *}$

\section{Affiliations:}

${ }^{1}$ Department of Chemistry, Purdue University; 560 Oval Dr., West Lafayette, IN 47907, USA.

${ }^{2}$ Purdue Center for Cancer Research; 201 S University St., West Lafayette, IN 47906, USA

${ }^{3}$ Purdue Institute of Inflammation, Immunology and Infectious Disease

${ }^{*}$ Corresponding author. Email: zhan2017@purdue.edu

\begin{abstract}
Precision control of molecular activities and chemical reactions in live cells is a long-sought capability by life scientists. No existing technology can probe molecular targets in cells and simultaneously control the activities of only these targets at high spatial precision and on the fly. We develop a real-time precision opto-control (RPOC) technology that detects a chemical-specific optical response from molecular targets during laser scanning and uses the optical signal to trigger an acousto-optic modulator, which allows a separate laser beam to only interact with the molecules of interest without affecting other parts of the sample. RPOC can automatically probe and control biomolecular activities and chemical processes in dynamic living samples with submicron spatial accuracy, nanoseconds response time, and high chemical specificity.
\end{abstract}




\section{Introduction}

The advancement of microscopy technologies has revealed unprecedented details of biological processes with superb resolution and chemical information. However, the capability to control chemical processes in live cells with high spatial accuracy and molecular selectivity in real-time is still lacking. Conventional chemical treatment by culturing cells with chemical compounds has poor spatial delivery selectivity and might pose off-target effects for the accurate understanding of compound-target interactions. Genetic methods such as CRISPR and RNA interference can control the expression and activity of proteins (1-3). However, transfection and incubation require sophisticated prepreparation and passaging processes with little temporal and spatial control. Optical tweezers and trapping can only physically manipulate a few pre-detected targets (4-6). Current laser ablation and manipulation methods are based on pre-image acquisition and manual operation of laser beams to interact with the target-of-interest (7-10). Optogenetics methods can control functions of neurons using light radiation and lightsensitive ion channels, however, require pre-imaging and demonstrate little sub-cellular precision (11-13). Thus, the existing optical manipulation technologies cannot apply to highly dynamic living biological samples to control molecular activities with high spatial accuracy and chemical specificity.

In this work, we develop a real-time precision opto-control (RPOC) technology that can detect and control molecules simultaneously, selectively, and precisely at the only desired activity sites. First, during laser scanning, an optical signal is generated at a specific pixel from target molecules. Then, the detected optical signal is compared with preset values using comparator circuitry. A desired optical signal will activate an acoustooptic modulator (AOM) which is used as a fast switch to couple another laser beam to interact at the same pixel. The optical signal detection, processing, and opto-control happen within $30 \mathrm{~ns}$ and in real-time during laser scanning. Digital logic functions allow opto-control of molecular activities based on the logic output from multiple signal channels. RPOC can accurately detect and control biomolecules in real-time without affecting other locations in the system. It is highly chemically selective since the optical signal can be selected from a range of responses such as fluorescence and Raman. This technology offers an unprecedented way to automatically and selectively control molecular activities and chemical reactions with sub-micron spatial precision.

\section{Results}

\section{The RPOC platform}

The concept of RPOC, which is based on fast laser scanning, is illustrated in Fig. 1, A and B. A laser(s) for optical signal excitation is scanning through the field of view. During the laser scanning, if a chemical-specific optical signal is detected and satisfies a preset condition (e.g. surpasses a threshold value), it will trigger an AOM to send a separate laser beam to interact with the sample at the same pixel in real-time. Optical signals that do not satisfy this condition will "turn off" the control laser beam to avoid laser interaction. Digital comparator circuits (fig. S1) were designed for presetting the selection conditions (e.g. the threshold $\mathrm{V}_{\mathrm{T}}$ ), performing analog/digital comparisons, and sending out a standard transistor-transistor logic (TTL) voltage for AOM control. A schematic of the RPOC system is shown in Fig. 1C. A dual-output femtosecond laser is used to perform 
optical signal excitation and opto-control. The $1045 \mathrm{~nm}$ laser output is used as the Stokes beam and the frequency-tunable laser output is used as the pump beam for stimulated Raman scattering (SRS) signal generation $(14,15)$. The laser beams are also used for two-photon excitation fluorescence (TPEF) signal excitation (16). Portions of both outputs are frequency-doubled to the visible range for opto-control. The selected control laser is sent to an AOM and commanded by the comparator circuits. The laser beam profiles after the AOM using ' 0 ' and '1' TTL commands are shown in Fig. 1, D and E, respectively. The $0^{\text {th }}$ order output is blocked by a beam stop and the $1^{\text {st }}$ order of the AOM output is combined with the excitation IR laser beams by a dichroic mirror before coupling into the microscope. Fig. 1F shows the $\sim 15$ ns response time of the comparator circuits. The AOM response time (Supplementary material) is calculated to be $\sim 7 \mathrm{~ns}$. Therefore, the response time of the opto-control system is less than $30 \mathrm{~ns}$, much shorter than the $10 \mu \mathrm{s}$ pixel dwell time for laser scanning. The spatial resolution of the SRS and TPEF modalities is measured to be $373 \mathrm{~nm}$ (fig. S2). The PROC control laser beam gives a spatial precision of $525 \mathrm{~nm}$ (fig. S3).

\section{Real-time control of active pixels (APXs) using chemical-specific optical signals}

An 'active pixel' (APX) is defined as the pixel location at which the control laser beam is turned on. Tracking APXs is critical for visualizing the opto-control locations. In the oversampling condition (the pixel size is smaller than the laser beam size at the focus), the size of the laser interaction area is larger than the size of the APXs (Fig. 2A). Higher intensity thresholds reduce APXs and laser interacting areas. Similarly, at the same intensity threshold, weaker optical signals above the threshold reduce APXs and the laser interaction areas (Fig. 2B). Increasing the pixel size might change these properties. Different optical signal intensities that would result in different APXs in the oversampling condition might give the same APXs and interaction areas (Fig. 2C). When the pixel size is greater than the actual laser beam size, the APXs might be the same or even larger than the actual interaction area (Fig. 2C). The oversampling condition is used throughout this work.

We first use fluorescence signals from microparticles to determine APXs. Laser pulses at $800 \mathrm{~nm}$ is used to excite the TPEF signals of mixed $1 \mu \mathrm{m}$ particles. Fig. 2D shows fluorescence signals detected in the 570/60 nm (left panel) and the 450/106 nm (right panel) fluorescence channels. The latter is paired with a comparator circuit box to determine the APXs. The selection condition is the fluorescence signal above a preset threshold $\mathrm{V}_{\mathrm{T}}$. When the $\mathrm{V}_{\mathrm{T}}$ is low $(0.5 \mathrm{~V}$ and $0.7 \mathrm{~V})$, APXs exceed the fluorescence pixels in the 450/106 $\mathrm{nm}$ channel (Fig. 2E). At a proper threshold $\mathrm{V}_{\mathrm{T}}=1.2 \mathrm{~V}$, APXs perfectly match the fluorescence signals. Fig. 2F compares the intensity profiles at selected lines in panels $A$ and $B$ when RPOC is turned off and on. The intensity increase is contributed by the leaking of the control laser which is only turned on by the fluorescence signal at the particles.

Fig. 2G shows chemical maps of mixed poly(methyl methacrylate) (PMMA) and polystyrene (PS) particles generated by hyperspectral SRS microscopy (17). Using Raman shifts at $2955 \mathrm{~cm}^{-1}$ or $3060 \mathrm{~cm}^{-1}$, we can determine APXs using either PMMA or PS SRS signals (Fig. $2 \mathrm{H}$ ). The selection condition is optical signals greater than $\mathrm{V}_{T}=1 \mathrm{~V}$. Fig. 2l displays the SRS spectra of PMMA and PS, the $\mathrm{V}_{T}$, and the selected Raman shifts 
for APX determination in Fig. 2E. APXs can be selected on different chemicals in realtime by tuning laser frequencies to match different Raman transitions (movie S1).

Using the lipid $\mathrm{CH}_{2}$ symmetric stretching SRS signals at $2855 \mathrm{~cm}^{-1}$, we can automatically select APXs only at the lipid droplets (LDs) in live cells, as shown in Fig. 2, J-L. Side-byside time-lapse images of SRS and APX selection using the lipid signals are shown in movie S2. The trajectory of APXs triggered by a single LD matches the corresponding LD trajectory (Fig. 2M, movie S3). We also demonstrate 3D precision controlling of the APXs in MIA PaCa-2 cells, as shown in movies S4 and S5. These results highlight the capability of tracing intercellular dynamics for APX determination using RPOC.

\section{Digital logic control of APXs}

A second comparator box with digital logic functions is also design as shown in fig. S4. digital logic functions can be selected from AND, OR, NAND, and NOR. Using the two comparator circuit boxes, we can select any intensity range from a single detector for APX determination. The connections to achieve this function are illustrated in Fig. 3A and fig. S5. Fig. 3B shows APXs selected on the LDs using only comparator box 1 and a single intensity threshold. Fig. 3C shows APXs determined using different intensity passbands between the upper and lower thresholds. The APXs selected between $\mathrm{V}_{\mathrm{T}}=0.2-0.3 \mathrm{~V}$ are more associated with the endoplasmic reticulum (ER) and between 0.1-0.16 V are mostly on cytosols. Spectral phasor analyses of hyperspectral SRS images of the same cells, which segment different cellular compartments (18), are shown in fig. S6 for comparison.

The connections for implementing the digital logic functions using two comparator boxes and two detectors are illustrated in fig. S7. The selection of the AND function is illustrated in Fig. 3D. We first demonstrate the AND function using mixed fluorescent PS particles, non-fluorescent PS particles, and nicotinamide adenine dinucleotide hydrogen (NADH) crystals, as shown in fig. S8 and S9. The AND function allows determining APXs only on the fluorescent PS particles that show up in both TPEF and SRS channels. Next, we used SRS to excite lipid signals (Fig. 3E) in MIA PaCa-2 cells and label the cells using a fluorescent ER tracker which can be visualized in the TPEF channel (Fig. 3F). By using an appropriate $\mathrm{V}_{\mathrm{T} 1}$ in the SRS channel and a low $\mathrm{V}_{\mathrm{T} 2}$ in the TPEF channel, APXs can be selected from most of the LDs in the cells (Fig. $\mathbf{3 G}$ ). Increasing the $\mathrm{V}_{\mathrm{T} 2}$ in the TPEF channel can exclude the LDs outside the ER and excite APXs on LDs only on the ER (Fig. 3H). These results demonstrate using the AND logic from two separate detectors for APX determination. The connections of OR, NAND, and NOR functions for RPOC are illustrated in fig. S10.

\section{Control and quantification of chemical changes at sub-micron precision}

To demonstrate precision control of chemical processes using the RPOC, we used a photochromic molecule, cis-1,2-dicyano1,2-bis(2,4,5-trimethyl-3-thienyl)ethene (CMTE), which can be changed from its open cis isomer (1a) to closed isomer (1) by UV light and switched back by visible light at $520 \mathrm{~nm}$ (Fig. 4A) (19, 20). A strong Raman signature peak at $1510 \mathrm{~cm}^{-1}$ can be detected for $\mathbf{1 b}$ but not $1 \mathbf{a}$ (19). The SRS signal from this peak can be visualized by tuning the pump beam to $902 \mathrm{~nm}$. We found that the combination of the pump and Stokes pulsed lasers for SRS imaging can also transform CMTE to 1b. A $522 \mathrm{~nm}$ laser beam frequency-doubled from the $1045 \mathrm{~nm}$ laser output is used for RPOC to convert $\mathbf{1 b}$ to $\mathbf{1 a}$ at selected subcellular locations. The Raman transition at $1510 \mathrm{~cm}^{-1}$ 
is used as the reporter for RPOC-induced $\mathbf{1 b}$ to $\mathbf{1 a}$ conversion during laser scanning (Fig. 4B).

First, we treat MIA PaCa-2 cells with CMTE and observed accumulation of CMTE in LDs of the cells due to the hydrophobic structure of the chemical (Fig. 4, C and D). Then, we used RPOC to convert $\mathbf{1 b}$ to $\mathbf{1 a}$ at selected locations of the sample with a control laser beam power of $\sim 10 \mu \mathrm{W}$. A single comparator box with a selection condition of $\mathrm{V}_{\mathrm{T}}>0.17 \mathrm{~V}$ was used to determine APXs which are majorly contributed by high-intensity CMTE aggregates, as shown in Fig. 4, C and D. After five frames of RPOC laser scanning, SRS signals at $1510 \mathrm{~cm}^{-1}$ from the APX-associated pixels are reduced, which can be visualized from the SRS intensity difference image in Fig. 4, C and D. If the control laser beam is constantly turned on during laser scanning for 20 frames, SRS signals at $1510 \mathrm{~cm}^{-1}$ on the entire image are significantly reduced (Fig. 4, C and D). The pixels where chemical conversion happened, as shown in the SRS intensity difference images, agree with the APXs. Fig. 4E plots SRS intensity profiles along the dashed lines in Fig. 4D, for images before and after the RPOC, the APXs, and after nonselective laser control after 20 frames. We see that laser-induced chemical changes of CMTE only happen at the APXs. Such chemical changes can be quantified by integrating the SRS intensity change of CMTE on APXs of each aggregate, which shows a quadratic dependence with the number of APXs from each aggregate (Fig. 4F). This nonlinear dependence arises from the oversampling condition used for RPOC, as illustrated in Fig. 2A. The mean SRS intensity change, on the other hand, has a near-linear dependence with the number of APXs (Fig. 4G). These analyses show that RPOC can not only selectively control chemical changes in space but also potentially quantify the amount of products and reaction rates.

\section{Precision control of molecular activities using different selection conditions}

RPOC can control CMTE at different parts of cells using various selection conditions. We first connected two comparator boxes as illustrated in fig. S3 to select an SRS signal range between two intensity levels. Here, the RPOC is only applied for a single-frame laser scanning with $10 \mu$ s dwell time per pixel. Fig. 5, A and B display the SRS signals from CMTE at $1510 \mathrm{~cm}^{-1}$ before the RPOC, the APXs, after a single frame RPOC, and the SRS intensity changes with a selection condition of $V_{L}=0.15 \mathrm{~V}$ and $V_{U}=0.4 \mathrm{~V}$, where $V_{L}$ and $V_{U}$ are the lower and upper signal limits for RPOC, respectively. The SRS image of $\mathrm{CH}_{2}$ stretching is shown in fig. S11. Since $\mathrm{Vu}$ is very high, this selection range determines the APXs mostly from the centers of the aggregates that contribute to strong CMTE SRS signals (Fig. 5C), similar to using a single comparator box as shown in Fig. 4. When the selection condition is chosen as $V_{L}=0.10 \mathrm{~V}$ and $\mathrm{VU}=0.12 \mathrm{~V}$, as shown in Fig. 5, D and E, weaker SRS signals selected APXs from the edges of most aggregates. In this case, the centers of the aggregates, which are not associated with APXs, are left unchanged, while the molecules at the edges of the aggregates are converted from $\mathbf{1 b}$ to 1a by RPOC. Fig. 5F plots the SRS intensity change and APXs along the dotted line and the intensity thresholds. The CMTE transition from $\mathbf{1 b}$ to $\mathbf{1 a}$ occurs only at the edges of the aggregates. There are pixels with noticeable SRS signal decrease outside APXs (Fig. $\mathbf{5 F}$ ). This is due to the use of an oversampling condition in which the RPOC laser interacting range is larger than the APXs, as illustrated in Fig. 2A.

Next, we used ROPC to selectively control the $\mathbf{1 b}$ to $\mathbf{1 a}$ conversion accumulated only in ER-associated LDs. SRS was used to detect CMTE targeting the $1510 \mathrm{~cm}^{-1}$ peak while 
ER tracker in the TPEF (550-600 nm) channel was used to delineate ER in live MIA PaCa2 cells. As shown in Fig. 6, A and B, APXs can be determined on ER-associated LDs using SRS and TPEF signals from two detectors with the digital AND function and appropriate threshold levels. The ER boundary is shown in Fig. 6B. By plotting the SRS intensity profiles of three LDs (Fig. 6C) and their corresponding APXs (Fig. 6D) along the dashed line (Fig. 6B), we find that LD \#3, which is not on the ER, is not affected by RPOC; while LDs \#1 and \#2, both on the ER, are converted from $\mathbf{1 b}$ to $\mathbf{1 a}$ by RPOC. These results demonstrate that digital logic RPOC can control molecular activities and chemical reactions associated with multiple organelles or related to organelle interactions.

\section{Discussion}

We for the first time demonstrate real-time precision opto-control of molecular activities and chemical processes triggered by optical signals from the molecules at submicron spatial precision. RPOC can perform active control of light-sensitive molecules and chemical reactions in living biological samples due to the fast response and automatic APX determination. In this work, we majorly focused on demonstrating the RPOC capability using a photoswitchable molecule CMTE. RPOC can also be applied to control newly developed photochromic vibrational probes $(21,22)$, widely used photoswitchable fluorescent molecules $(23,24)$, and light-sensitive chemical reactions $(25-28)$ at high spatial and temporal accuracy.

The continuous improvement of RPOC will lead to more opportunities in biophotonics and biological sciences. For example, further optimization of the control laser beam can improve the RPOC precision. Instead of using an expensive femtosecond laser, a more cost-effective and compact RPOC platform can be developed based on continuous-wave (CW) lasers. The CW-RPOC system would greatly reduce the system cost and ideal for integration with commercial fluorescence microscopes. Programmable acousto-optic tunable filters would also allow for the selection of different laser beams automatically for RPOC. Improvement in optics and electronics, such as using an electro-optic modulator and resonant mirrors would further improve the RPOC response time for high-speed laser scanning systems.

PROC offers a way for biologists and chemists to control biomolecular behaviors and chemical reactions precisely and automatically in space and time without affecting unwanted targets. We believe RPOC will have important applications, when combined with photoactivatable molecules, for better control of enzyme activities, high accuracycontrolled release, high precision optogenetics, and improved precision treatment. Applying digital logic functions in RPOC with photoswitchable fluorescent molecules would also enable recording and saving organelle interactions for live systems. Future research will focus on demonstrating the capabilities of RPOC in these applications.

\section{References}

1. R. Y. Tsien, The green fluorescent protein, Annu. Rev. Biochem., 67, 509 (1998).

2. P. J. Paddison, A. A. Caudy, E. Bernstein, G. J. Hannon, D. S. Conklin, Short hairpin RNAs (shRNAs) induce sequence-specific silencing in mammalian cells, Genes Dev., 16, 948 (2002). 
3. R. Barrangou et al., CRISPR provides acquired resistance against viruses in prokaryotes, Science, 315, 1709 (2007).

4. J. Enger, M. Goksör, K. Ramser, P. Hagberg, D. Hanstorp, Optical tweezers applied to a microfluidic system, Lab. Chip, 4, 196 (2004).

5. H. Zhang, K.-K. Liu, Optical tweezers for single cells, J. R. Soc. Interface, 5, 671 (2008).

6. M. Daly, M. Sergides, S. Nic Chormaic, Optical trapping and manipulation of micrometer and submicrometer particles, Laser Photonics Rev., 9, 309 (2015).

7. S. A. Boppart et al., High-Resolution Optical Coherence Tomography-Guided Laser Ablation of Surgical Tissue, J. Surg. Res., 82, 275 (1999).

8. $\mathrm{H}$. He et al., Manipulation of cellular light from green fluorescent protein by a femtosecond laser, Nat. Photonics, 6, 651 (2012).

9. F. Shi et al., Mitochondrial swelling and restorable fragmentation stimulated by femtosecond laser, Biomed. Opt. Express, 6, 4539 (2015).

10. T. Meyer et al., CARS-imaging guidance for fs-laser ablation precision surgery, Analyst, 144, 7310 (2019).

11. K. Deisseroth et al., Next-Generation Optical Technologies for Illuminating Genetically Targeted Brain Circuits, J. Neurosci., 26, 10380 (2006).

12. A. R. Adamantidis, F. Zhang, A. M. Aravanis, K. Deisseroth, L. de Lecea, Neural substrates of awakening probed with optogenetic control of hypocretin neurons, Nature, 450, 420 (2007).

13. E. Papagiakoumou et al., Scanless two-photon excitation of channelrhodopsin-2, Nat. Methods, 7, 848 (2010).

14. C. W. Freudiger et al., Label-free biomedical imaging with high sensitivity by stimulated Raman scattering microscopy, Science, 322, 1857 (2008).

15. J.-X. Cheng, X. S. Xie, Vibrational spectroscopic imaging of living systems: An emerging platform for biology and medicine, Science, 350, (2015).

16. P. T. So, C. Y. Dong, B. R. Masters, K. M. Berland, Two-photon excitation fluorescence microscopy, Annu. Rev. Biomed. Eng., 2, 399 (2000).

17. D. Fu, G. Holtom, C. Freudiger, X. Zhang, X. S. Xie, Hyperspectral imaging with stimulated Raman scattering by chirped femtosecond lasers, J. Phys. Chem. B, 117, 4634 (2013).

18. D. Fu, X. S. Xie, Reliable cell segmentation based on spectral phasor analysis of hyperspectral stimulated Raman scattering imaging data, Anal. Chem., 86 9, 4115 (2014).

19. J. Shou, Y. Ozeki, Photoswitchable stimulated Raman scattering spectroscopy and microscopy, Opt. Lett., 46, 2176 (2021).

20. F. G. Erko et al., Spectral, Conformational and Photochemical Analyses of Photochromic Dithienylethene: cis-1, 2-Dicyano-1, 2-bis (2, 4, 5-trimethyl-3thienyl) ethene Revisited, Eur. J. Org. Chem., 2013, 7809 (2013).

21. J. Du, L. Wei, Multicolor Photoactivatable Raman Probes for Subcellular Imaging and Tracking by Cyclopropenone Caging, J. Am. Chem. Soc., (2021).

22. J. Ao et al., Switchable stimulated Raman scattering microscopy with photochromic vibrational probes, Nat. Commun., 12, 1 (2021).

23. S. Habuchi et al., Reversible single-molecule photoswitching in the GFP-like fluorescent protein Dronpa, Proc. Natl. Acad. Sci. U. S. A., 102, 9511 (2005). 
24. R. Ando, H. Hama, M. Yamamoto-Hino, H. Mizuno, A. Miyawaki, An optical marker based on the UV-induced green-to-red photoconversion of a fluorescent protein, Proc. Natl. Acad. Sci. U. S. A., 99, 12651 (2002).

25. X. Chen et al., Acetylcholinesterase inhibitors with photoswitchable inhibition of $\beta$ amyloid aggregation, ACS Chem. Neurosci., 5, 377 (2014).

26. M. Borowiak et al., Photoswitchable inhibitors of microtubule dynamics optically control mitosis and cell death, Cell, 162, 403 (2015).

27. S. Mondal, S. S. Parelkar, M. Nagar, P. R. Thompson, Photochemical control of protein arginine deiminase (PAD) activity, ACS Chem. Biol., 13, 1057 (2018).

28. B. A. Copits et al., A photoswitchable GPCR-based opsin for presynaptic inhibition, Neuron, 109, 1791 (2021).

\section{Author contributions:}

C.Z. designed the project and experiment. C.Z. and M.G.C. performed the experiments, analyzed the results, and wrote the paper. M.G.C. constructed the optical system for RPOC. G.A.G. helped prepare the cancer cells for imaging. J.A.M helped in biological sample preparation. M.S.C. designed and fabricated the comparator circuits. G.E. designed and fabricated the tuned amplifier and photodetector for SRS signal detection.

Competing interests: Authors declare that they have no competing interests.

Data and materials availability: All data, code, and materials used in the analysis are available in the corresponding authors' lab. All data are available in the main text or the supplementary materials. 


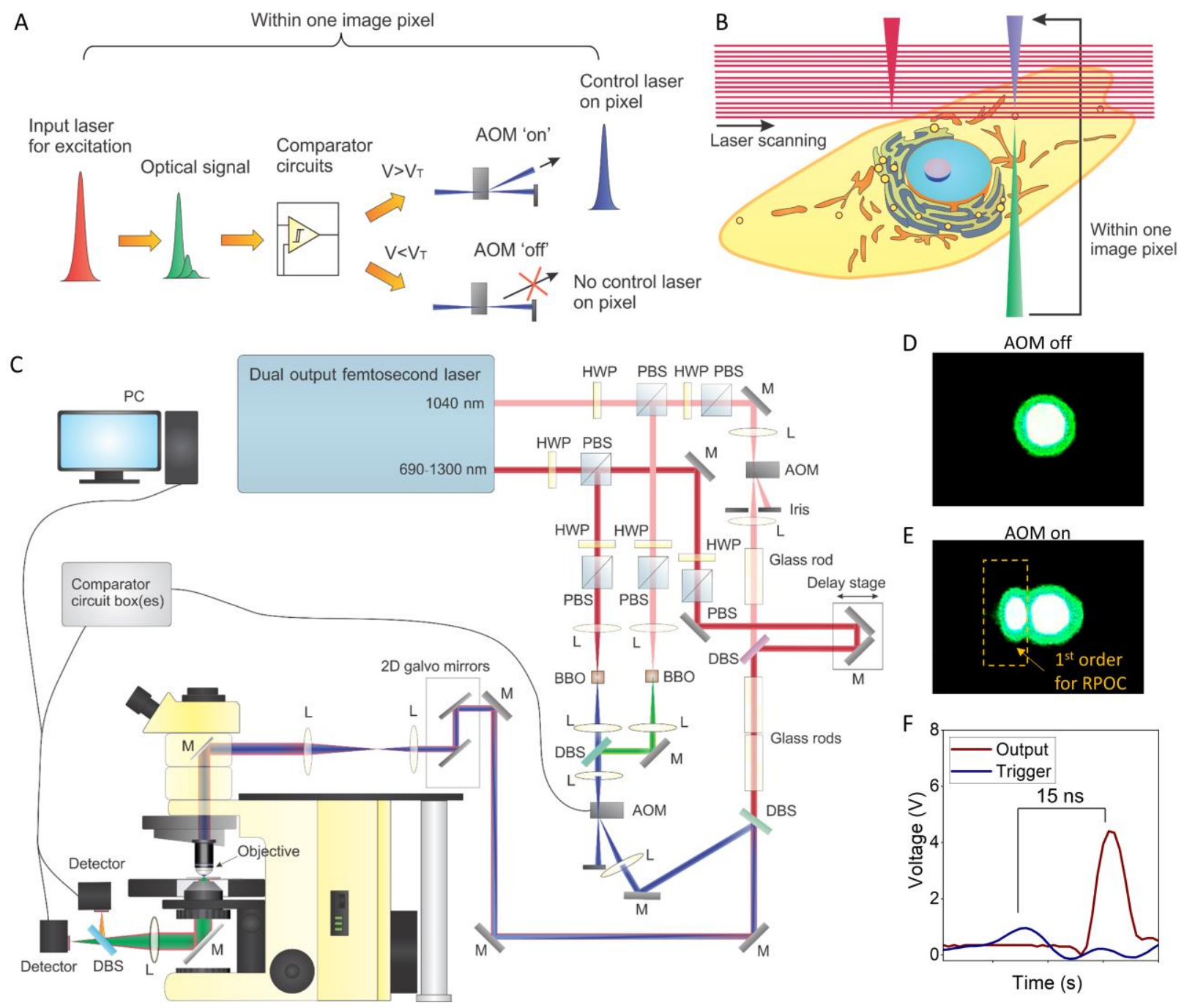

Fig. 1. The RPOC concept and optical configuration. (A) An illustration of the RPOC concept. (B) The illustration of RPOC for selective control of molecular activities in a cell during laser scanning. (C) A schematic of the RPOC experimental setup. HWP, half-wave plate; L, lens; M, mirror; DBS, dichroic beam splitter; PBS, polarization beam splitter; AOM, acousto-optic modulator. (D) The profile of the control laser beam (at $522 \mathrm{~nm}$ ) after the AOM when the AOM is turned off. (E) The profile of the control laser beam after the $A O M$ when the $A O M$ is turned on (the $1^{\text {st }}$ order deflection is highlighted). (F) The response time of the comparator box is measured to be $\sim 15 \mathrm{~ns}$. 


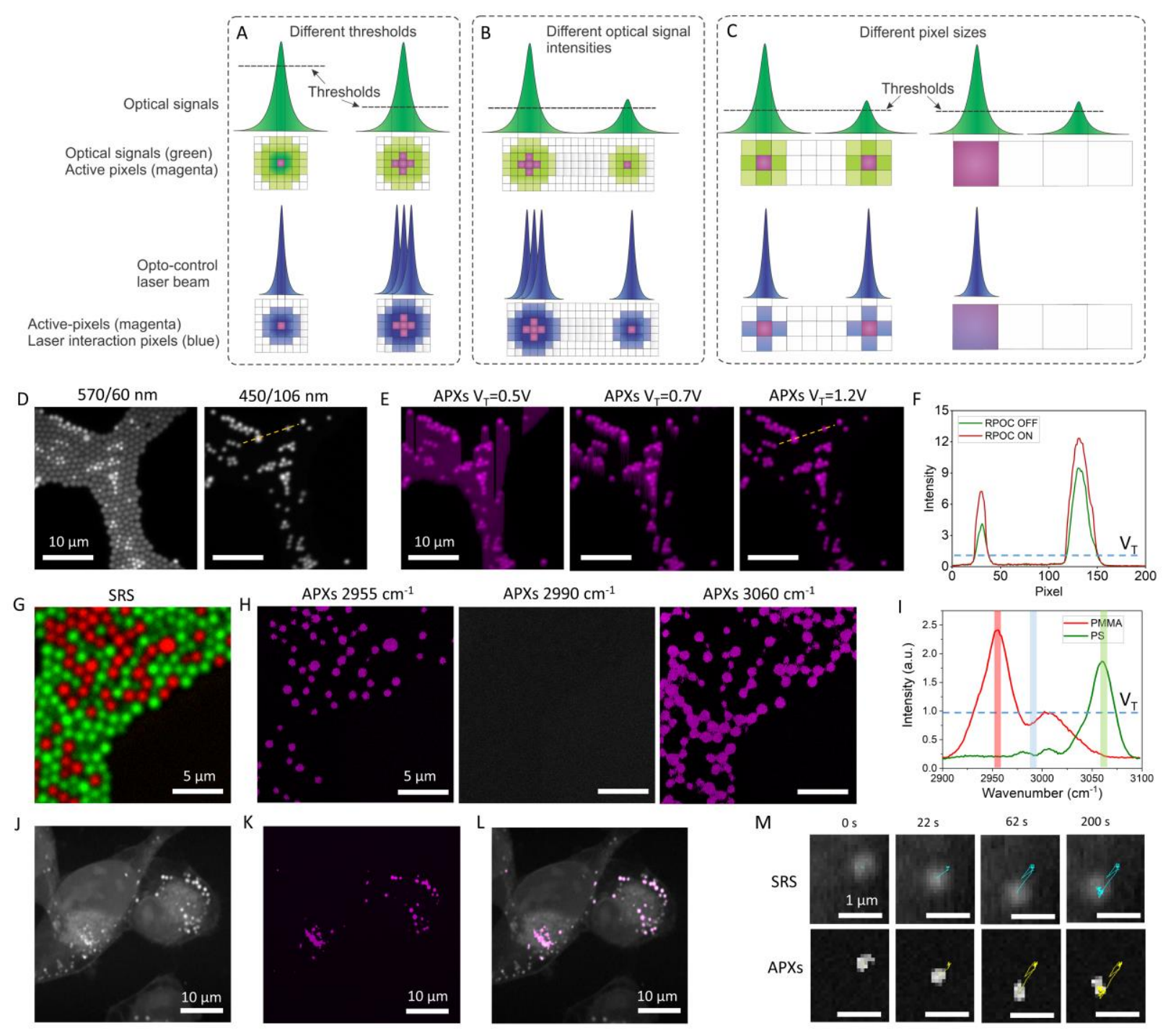

Fig. 2. Mapping out RPOC APXs. (A) An illustration of APX selection using different signal thresholds in the over-sampling condition. The green pulses and pixels indicate optical signals, the magenta pixels indicate APXs, and the blue pulses and pixels indicate the interaction pulses and pixels. (B) An illustration of APX selection using different optical intensities in the over-sampling condition. (C) An illustration of APX selection at larger pixel sizes for different optical signals. (D) Mixed fluorescent particles detected in the $570 / 60 \mathrm{~nm}$ channel (left, orange and green fluorescent particles) and 450/106 nm channel (right, only the green fluorescent particles). (E) APXs determined using different $V_{T}$ values for the signals from the 450/106 nm channel. (F) Comparing the 450/106 nm channel optical intensity when the RPOC is turned on (red) and off (green). (G) A pseudo-color SRS image containing PMMA (red) and PS (green) particles. (H) APXs determined using the PS peak at $2950 \mathrm{~cm}^{-1}$ (left), PMMA peak at $3060 \mathrm{~cm}^{-1}$ (right), and no Raman peaks at $2990 \mathrm{~cm}^{-1}$ (middle). (I) SRS spectra of PMMA (red) and PS (green). The red, green, and blue lines are wavenumbers used for RPOC in panel H. (J) An SRS image of MIA $\mathrm{PaCa}-2$ cells in the lipid $\mathrm{CH}_{2}$ stretching region. (K) APXs determined using SRS signals from LDs. (L) An overlay of the SRS image and the APXs turned on only at the LDs. (M) 
Time-lapse SRS images of a LD in a live MIA PaCa-2 cell (top row) and the corresponding APXs determined by the SRS signals (bottom row). The color curves plot trajectories of the LD and the APXs in $200 \mathrm{~s}$.
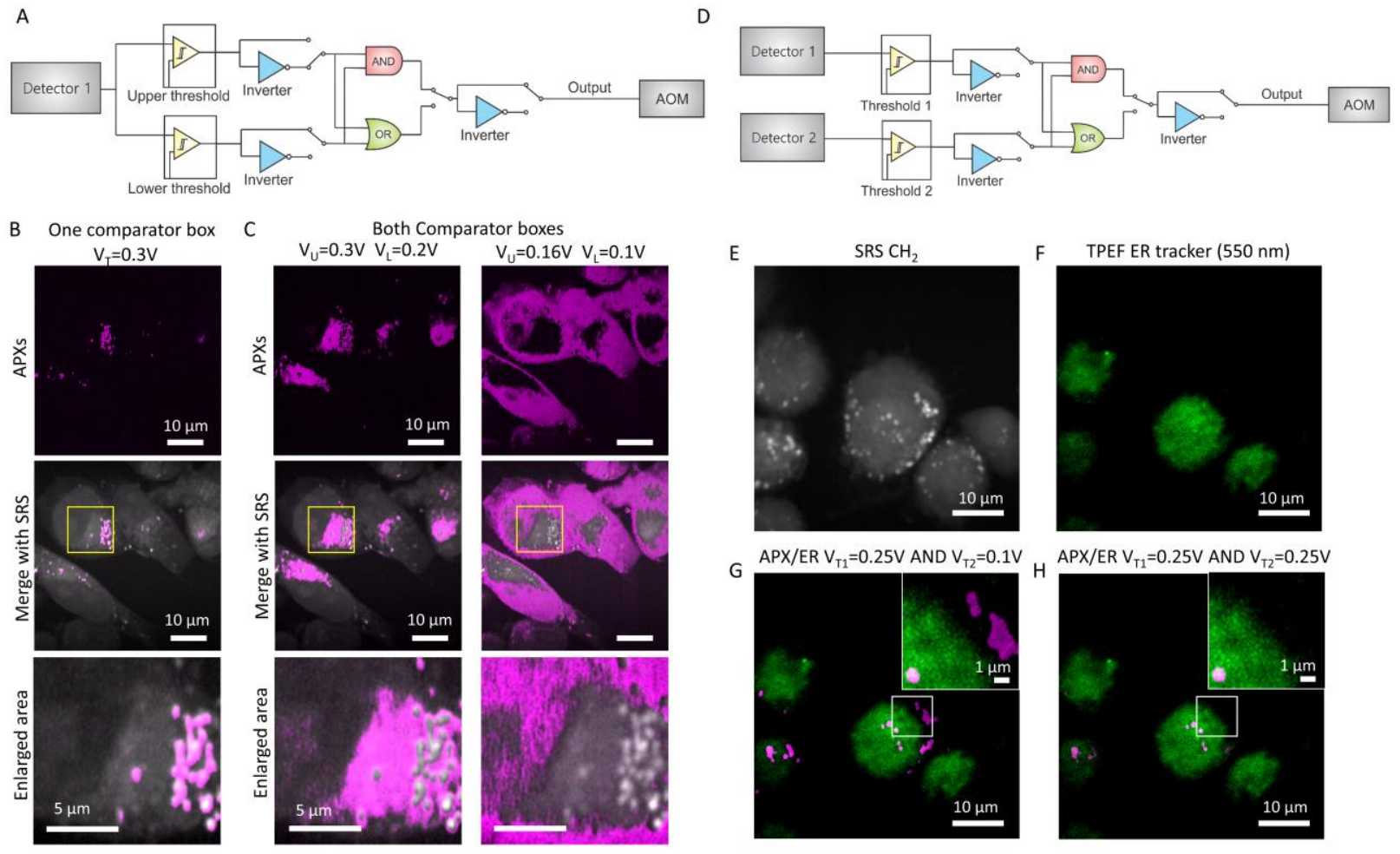

Fig. 3. Digital logic control of APXs. (A) Illustration of electronics to select an intensity passband for determination of APXs. (B) The APXs selected using only one comparator box with $\mathrm{V}_{\mathrm{T}}=0.3 \mathrm{~V}$ (upper panel), the overlay of the APXs with the corresponding SRS image from the same field of view (middle panel), and a magnified image from the selected area (bottom panel). (C) APXs selected using two comparator boxes with the intensity range between 0.2-0.3 $\mathrm{V}$ (left panels) and 0.1-0.16 V (right panels). (D) Illustration of electronics to choose the AND function for determination of APXs using two comparator boxes. (E) An SRS image of MIA PaCa-2 cells at the $\mathrm{CH}_{2}$ stretching vibration. (F) An TPEF image of the MIA PaCa-2 cells labeled using ER tracker. (G) An overlay of the TPEF image from $E R$ and the APXs determined using $V_{T 1}=0.25 \mathrm{~V}$ (SRS) and $\mathrm{V}_{\mathrm{T} 2}=0.25$ $\mathrm{V}$ (TPEF). (H) An overlay of the TPEF image from ER and the APXs using $\mathrm{V}_{\mathrm{T} 1}=0.25 \mathrm{~V}$ (SRS) and $\mathrm{V}_{\mathrm{T} 2}=0.25 \mathrm{~V}$ (TPEF). 
A

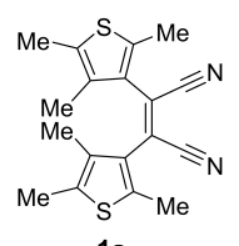

1a

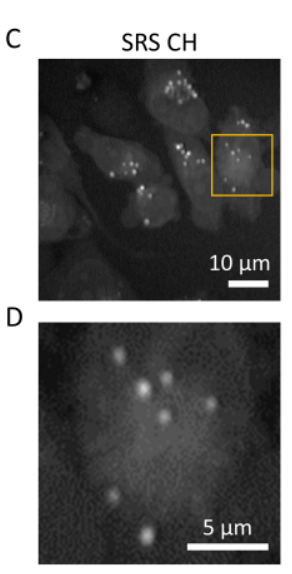

E
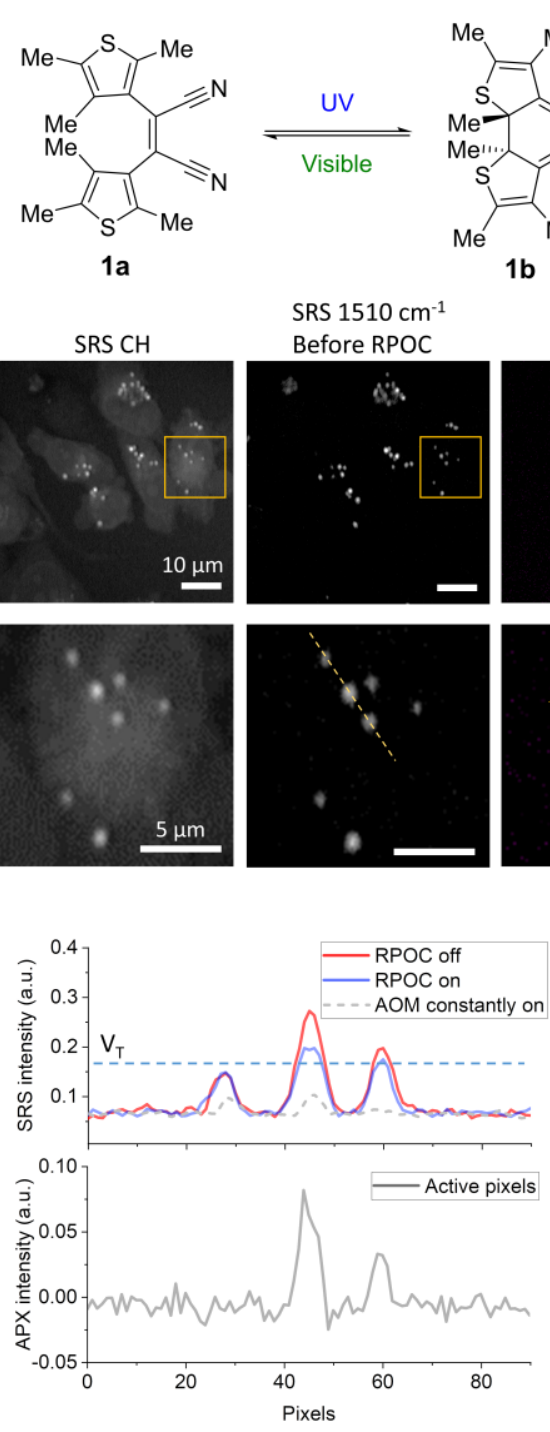

SRS $1510 \mathrm{~cm}^{-1}$ Before RPOC
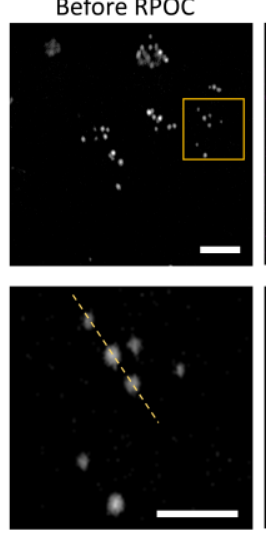

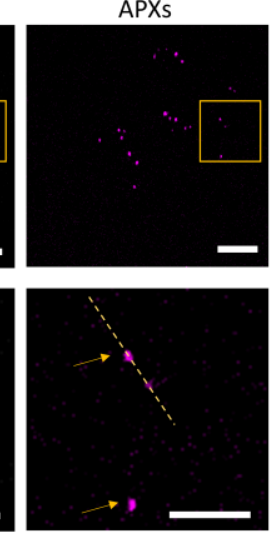

$\mathrm{F}$
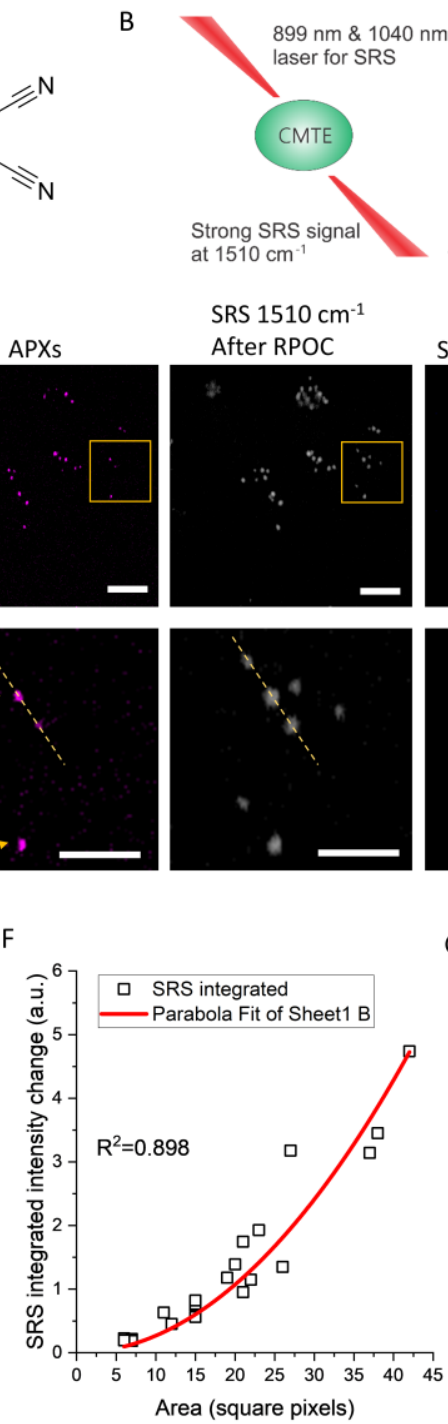

at $1510 \mathrm{~cm}^{-1}$

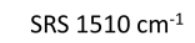

After RPOC
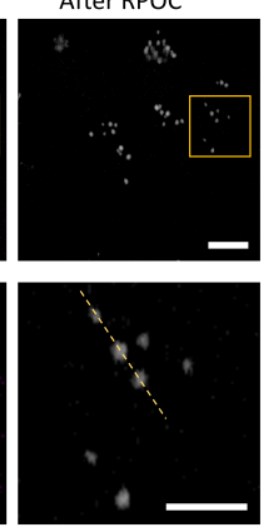

B
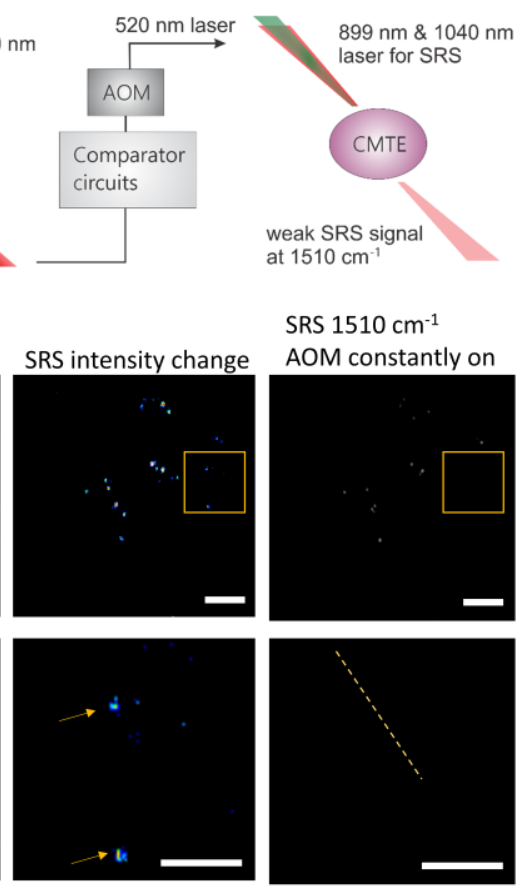

SRS $1510 \mathrm{~cm}^{-1}$ AOM constantly on

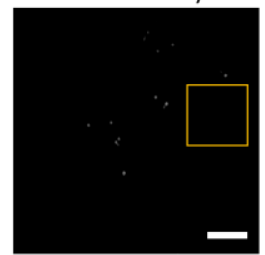

G

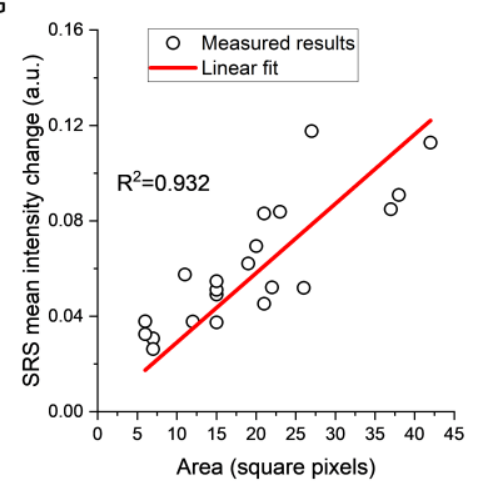

Fig. 4. Precision control and quantitative comparison of site-specific chemical changes using RPOC. (A) An illustration of switching states of CMTE between the open cis isomer (1a) and the closed isomer (1b) forms using UV and visible (green) light. (B) An illustration of the workflow for CMTE conversion by the RPOC using $1510 \mathrm{~cm}^{-1}$ SRS signal and a $522 \mathrm{~nm}$ laser. (C) An SRS image in the $\mathrm{CH}_{2}$ region, at $1510 \mathrm{~cm}^{-1}$ before RPOC, the APXs, at $1510 \mathrm{~cm}^{-1}$ after RPOC using $\mathrm{V}_{T}=0.17 \mathrm{~V}$, the SRS intensity difference before and after RPOC, at $1510 \mathrm{~cm}^{-1}$ after the AOM constantly on for 20 frames. (D) Magnified images of the selected areas in panel C. (E) SRS intensity profiles of images and APXs long the dotted lines in panel D. The dashed curve shows the SRS intensity profile along the same line after 20 scan frames with AOM constantly on. (F) Integrated SRS intensity changes of CMTE as a function of the number of APXs for CMTE aggregates. Open circles are experimental results, the curve is the quadratic fitting. (G) Mean SRS intensity changes of CMTE as a function of the number of APXs for CMTE aggregates. Open circles are experimental results, the line is the linear fitting. 

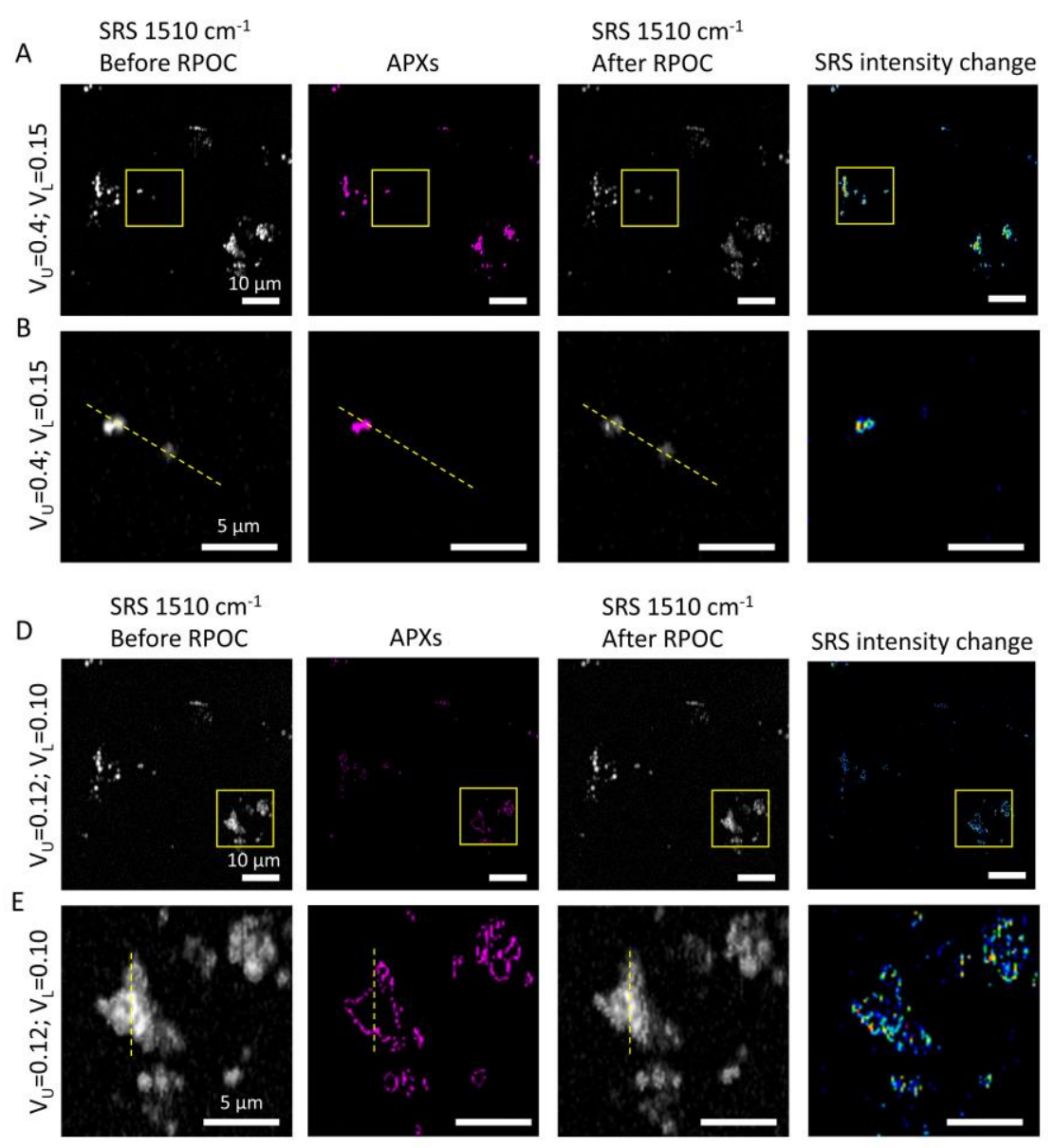

SRS $1510 \mathrm{~cm}^{-1}$
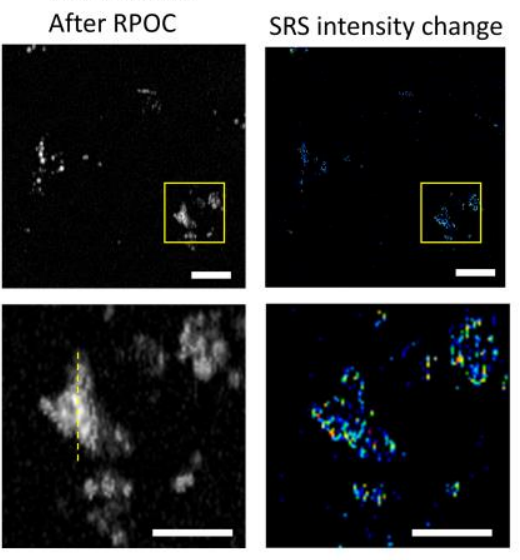
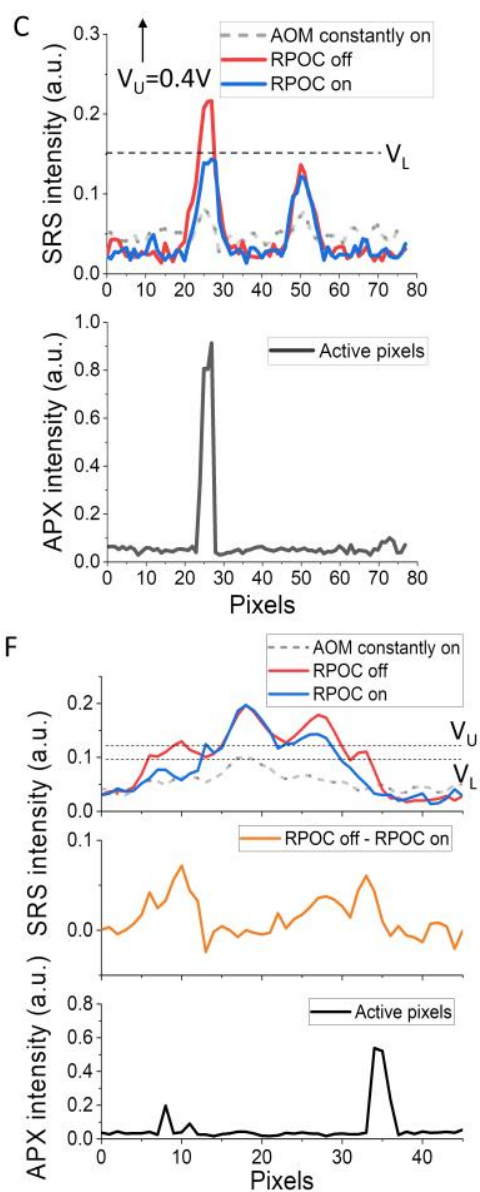

Fig. 5. Precision control of chemical changes by RPOC using different optical intensity ranges. (A) An SRS image at $1510 \mathrm{~cm}^{-1}$ before RPOC (left), APXs selected by an upper threshold $\left(\mathrm{V}_{\mathrm{U}}\right)$ of $0.4 \mathrm{~V}$ and lower threshold $\left(\mathrm{V}_{\mathrm{L}}\right)$ of $0.15 \mathrm{~V}$ (middle left), an SRS image at $1510 \mathrm{~cm}^{-1}$ after the RPOC (middle right), and the SRS intensity difference before and after the RPOC (right). (B) Magnified images from the highlighted regions in panel A. (C) SRS intensity profiles of images long the dotted lines in panel B. The dashed curve shows the SRS intensity profile along the same line after 20 scan frames with AOM constantly on. (D) Similar images as in panels $A$, using $V_{U}=0.12 \mathrm{~V}$ and $\mathrm{V} L=0.10 \mathrm{~V}$ for $A P X$ selection. (E) Magnified images from the selected regions in panel D. (F) SRS intensity profiles of images long the dotted lines in panel $\mathrm{E}$. The dashed curve shows the SRS intensity profile along the same line after 20 scan frames with AOM constantly on. The middle panel plots SRS intensity difference before and after RPOC. 

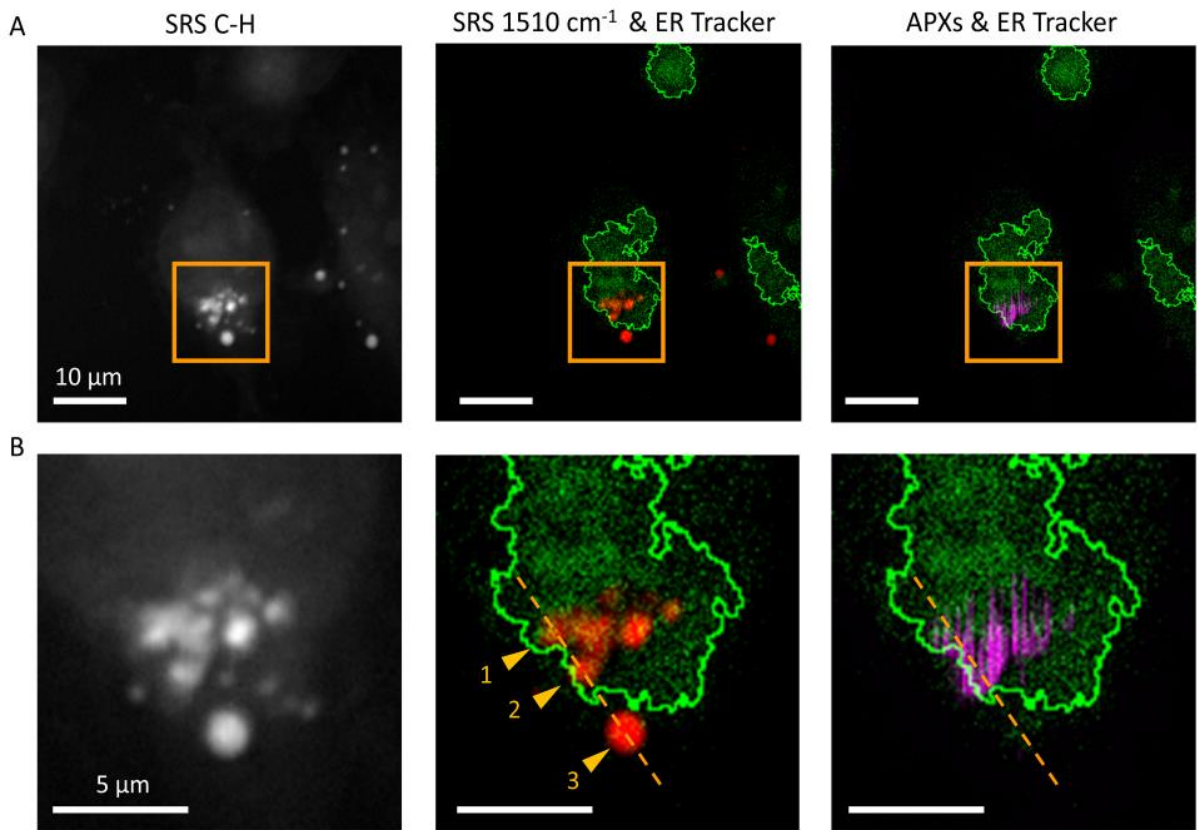

SRS intensity change at $1510 \mathrm{~cm}^{-1}$

C
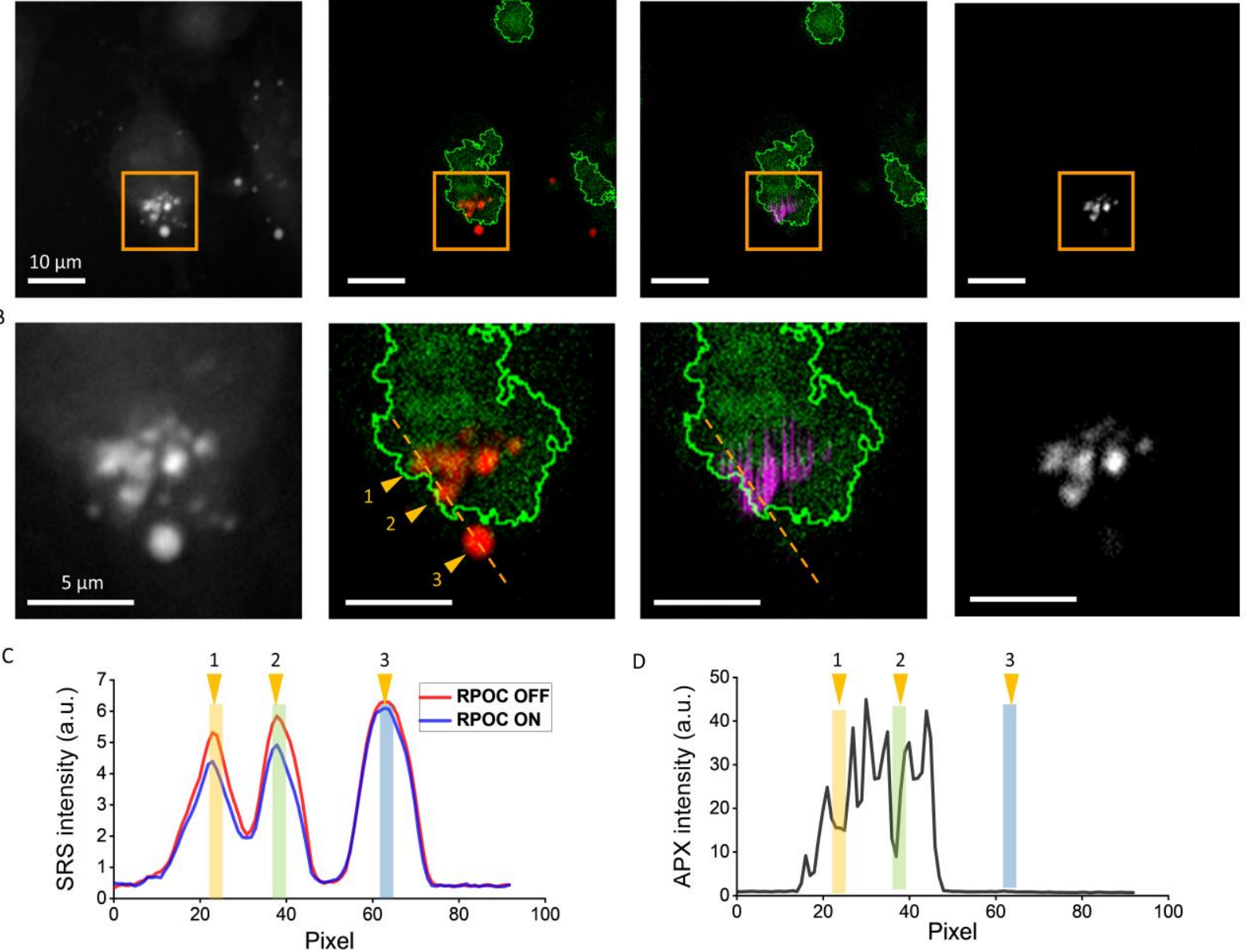

Fig. 6. Precision control of chemical changes by RPOC using digital logic from two detectors. (A) Images of MIA PaCa-2 cells showing SRS signals at $2855 \mathrm{~cm}^{-1} \mathrm{CH}$ stretching, the overlay of SRS signals from CMTE at $1510 \mathrm{~cm}^{-1}$ and ER tracker fluorescence signals, the overlay of APXs and ER tracker signals, and the CMTE SRS signal changes after RPOC. (B) Magnified images from the highlighted areas in panel A. (C) CMTE SRS intensity profiles before and after RPOC along the selected lines in panel B. (D) Intensity profiles of APXs. Colored bars highlight positions of selected LDs in panel B. 